\title{
Engagement académico
}

\author{
Academic engagement \\ Ángel D. Amador-Pérez ${ }^{a}$, Angélica Romero Palencia ${ }^{b}$, Rebeca Ma. Elena Guzmán Saldaña ${ }^{c}$
}

\begin{abstract}
:
This article derived from a theoretical review describes how academic engagement arises from the need to increase the performance of students in the school environment, specifically at the college. At the beginning, this concept was applied only for organizations, initially considering it as the opposite of burnout and subsequently under its own theoretical reference. The main theoretical models that support this variable are expressed, as well as the main scales for its measurement.
\end{abstract}

Keywords:

Engagement, students, burnout

\section{Resumen:}

El presente trabajo derivado de una revisión teórica se describe cómo el engagement académico surge ante la necesidad de incrementar el desempeño de los estudiantes en el ámbito escolar, específicamente en la universidad. Con anterioridad este concepto era aplicado únicamente para el ámbito de las organizaciones, en un principio considerándole como la parte contraria del desgaste laboral (burnout) y posteriormente bajo su propio referente teórico. Se expresan los principales modelos teóricos que fundamentan a esta variable, así como las principales escalas para su medición.

Palabras Clave:

Engagement, estudiantes, burnout

\section{Introducción}

Durante mucho tiempo, la comunidad estudiantil universitaria ha experimentado diversas dificultades en torno a: la falta de motivación por continuar estudiando, el compromiso dedicado a los estudios, la carrera de elección, e incluso a tener que trabajar para poder solventar los estudios, o diversos motivos que afectan a los alumnos y que puede llegar a convertirse en lo que conocemos cómo síndrome del desgaste o "burnout", algo que antes era aplicado únicamente en el ámbito organizacional, para empleados y trabajadores, pero que actualmente se ha detectado que puede aparecer en otros ámbitos como el estudiantil(1). Por tanto, surge la necesidad de detectar constructos que ayuden a la motivación, al compromiso y a la dedicación desde una perspectiva positiva, relacionándose con la percepción de bienestar en el estudiante (2).

Así, surge el constructo "engagement", inicialmente, como la contraparte positiva del burnout, de tal manera que, aplicado en la educación, el engagement académico permite detectar y medir que tan involucrados están los estudiantes con lo que aprenden y viven dentro del ámbito escolar y cómo aprenden a desenvolverse en la vida estudiantil (3).

\section{El engagement en el ámbito escolar}

En las últimas dos décadas se ha empleado el anglicismo engagement para denominar a un constructo psicológico que define un estado mental específico, objetivable mediante escalas particularmente diseñadas y validadas, asociado con el grado de involucramiento positivo de las personas con sus estudios. A la fecha no tiene una traducción exacta al español, que pueda transmitir de manera fidedigna su significado (2).

El engagement académico es un estado positivo y afectivocognitivo compuesto por tres elementos: vigor, dedicación y absorción (1).

A pesar de que el estudio del engagement tiene sus inicios en el ámbito de las organizaciones, el interés hacia el análisis de este constructo se ha trasladado al ámbito educativo ya que sugiere la experiencia por parte de los estudiantes de sensaciones que hacen que estos muestren un mayor grado de implicación con

\footnotetext{
a, Universidad Autónoma del Estado de Hidalgo, Instituto de Ciencias de la Salud Hidalgo, Email: adavidntoledo@gmail.com

${ }^{\text {b }}$ Autor de Correspondencia, Universidad Autónoma del Estado de Hidalgo, Instituto de Ciencias de la Salud, ORCID: 0000-0003-09336937, Email: angelica_romero@uaeh.edu.mx

${ }^{c}$ Universidad Autónoma del Estado de Hidalgo, Instituto de Ciencias de la Salud Hidalgo, ORCID: 0000-0003-0877-4871, Email: remar64@yahoo.com.mx
} 
sus estudios. De ahí que, en la actualidad, el engagement académico se asocie con el rendimiento y el bienestar de los estudiantes (2).

Diferentes autores $(2,3)$ abogan por un modelo de dos dimensiones del engagement: conductual y psicológica. La dimensión conductual involucra comportamientos tales como buscar consejo en los docentes, dedicar tiempo al estudio fuera de clase y adoptar un enfoque profundo de aprendizaje, mientras que la dimensión psicológica involucra la orientación académica de los alumnos, sobre todo el valor que los mismos asignan al esfuerzo académico y al aprendizaje.

Un modelo alternativo del engagement sugiere dos dimensiones distintas: el engagement conductual y el emocional (3). Desde esta perspectiva el engagement conductual se refiere a la participación activa en el proceso de aprendizaje y se operacionaliza en función del esfuerzo y persistencia que invierte el estudiante, mientras que el engagement emocional involucra las reacciones afectivas experimentadas durante las actividades académicas. Esta dimensión involucraría emociones positivas y emociones negativas.

En ambos casos el engagement se ha delimitado a partir de diferentes modelos teóricos cuyo origen se remonta al desempeño de los trabajadores en sus entornos laborales, como se verá a continuación.

\section{Modelo JD-R (Job Demands-Resources Model; Modelo de Demandas Recursos Laborales)}

Este modelo fue introducido por Demerouti, Bakker, Nachreiner y Schaufeli y se enfoca primeramente en los aspectos de trabajo que son fuente de sensaciones estresantes y agotamiento para después examinar los aspectos que ayudan a tener éxito en el trabajo, incluso en circunstancias estresantes: elementos que permiten al trabajador sentirse con energía, proveer sensación de orgullo y propósito.

El modelo plantea que algunos aspectos estresantes del trabajo consisten en la falta de recursos necesarios para las tareas requeridas. Así, se incluyen dos conjuntos específicos de condiciones de trabajo para la predicción del bienestar del empleado: a) las demandas laborales: aspectos psicológicos, sociales $\mathrm{u}$ organizacionales del trabajo que requieren un esfuerzo físico o mental prolongado, lo que se asocia con costos fisiológicos y psicológicos, como el agotamiento y b) los recursos laborales: aspectos físicos, psicológicos, sociales u organizacionales que pueden propiciar aspectos positivos tales como, ser funcional logrando metas del trabajo, reducir demandas laborales asociadas a costos fisiológicos y psicológicos y estimular el crecimiento personal y el desempeño (4).

\section{Modelo RED}

El modelo de Recursos-Experiencia-Demandas surge como una extensión del modelo de Demandas-Recursos laborales. Este modelo toma en cuenta las variables: a) las demandas-recursos laborales, b) los recursos personales y c) las emocionesexperiencias y conductas laborales.

En cuanto a las demandas y recursos laborales, además de contemplar lo planteado en el modelo JD-R, está dimensión hace una diferenciación en las demandas laborales y las distingue en "demandas amenazantes" y "demandas retadoras" (4).

Las demandas amenazantes son aquellas demandas negativas que potencialmente pueden dañar los beneficios y logros personales, provocando emociones negativas y fomentan el afrontamiento emocional. Favorecen el bajo desempeño, insatisfacción, carencia de motivación abandono del trabajo.

Las demandas retadoras: son valoradas de forma positiva, puesto que, tienen el potencial de promover los logros personales, favoreciendo a un enfrentamiento basado en la solución de problemas. Se asocian con el rendimiento, motivación, satisfacción, emociones y actitudes positivas al trabajo. (4)

En lo que respecta a los recursos personales, estos autores, se refieren a los aspectos personales que motivan, permiten el aprendizaje y fomentan el desarrollo individual y colectivo, así como, permiten afrontar las demandas laborales, solas o en conjunto con los recursos laborales(4).

\section{Modelo HERO}

El modelo de Organizaciones Saludables y Resilientes (HEalthy \& Resilient Organizations-HERO) postula que los recursos laborales están relacionados con la salud psicosocial de los empleados, mejorando su calidad de vida y está conformado por tres elementos:

a) Los recursos y prácticas laborables saludables, que permiten dar estructura y gestión a los procesos de trabajo influyentes al aparecer, b) El capital psicológico positivo y c) los resultados laborales sanos.

Esta propuesta plantea que las prácticas saludables influyen en el desarrollo de equipos de trabajo, resultados laborales, estructura y organización de procesos de trabajo provocando una mejora que se mantiene en el tiempo.

Este modelo brinda gran atención al bienestar psicosocial dentro de un grupo, en concreto con el engagement grupal, permitiendo aprovechar los recursos sociales de la organización, incluyendo como uno de los recursos más importantes, el liderazgo transformacional, que conduce hacia un fin compartido por todo el equipo, obteniendo el compromiso del mismo y la organización (4).

Estos mismos modelos se han incluido como la base del engagement académico y se han empleado para explicar el fenómeno.

Adicionalmente a los modelos explicativos, existen una serie de instrumentos que han sido empleados para medir el engagenment como se describirá a continuación. 


\section{Utrecht Work Engagement Scale (UWES-S)}

En un principio, la escala estaba formada por 24 afirmaciones que en su mayoría eran frases del Inventario de Burnout de Maslach escritas en positivo. Con esta escala se realizaron análisis psicométricos en una muestra de empleados y una de estudiantes, resultando siete afirmaciones inconsistentes que fueron eliminadas, quedando constituida una segunda versión UWES-17 con 17 ítems, que incluía seis afirmaciones de vigor, cinco de dedicación y seis de absorción, con valores de confiablidad de entre 0.78 y 0.79 , Sin embargo un nuevo análisis derivado de una aplicación posterior, encontró dos afirmaciones más que resultaron inconsistentes, por lo que algunos estudios eliminan estas afirmaciones y utilizan la versión UWES-15, formados por las cinco afirmaciones de mayor peso para cada factor. Esta última versión también evalúa los tres elementos que integran el engagement: vigor, dedicación y absorción, con 15 afirmaciones en escala de Likert, desde 0 (Nunca) hasta 6 (Siempre) (4).

Aplicaciones en las que emplearon estas escalas en empleados encontraron que el engagement es un recurso importante para generar satisfacción y bienestar psicológico en los empleados. (5).

La versión de la escala diseñada para estudiantes (UWES-S) validada para México, evalúa los tres elementos que integran el engagement: vigor dedicación y absorción, con 15 afirmaciones en escala de Likert, desde 0 (Nunca) hasta 6 (siempre). El Alpha de Crombach total es de 0.88 (11).

En la actualidad, el engagement académico se asocia con el rendimiento y el bienestar de los estudiantes (7). Desde este enfoque positivo, hay autores que indican que un mayor grado de engagement perfila estudiantes con actitudes más entusiastas y optimistas hacia el aprendizaje (8).

Es importante recalcar, que la mayoría de los estudios se han enfocado al ámbito laboral, realizando las aplicaciones en trabajadores únicamente. Es por eso que la versión UWES-S, diseñada exclusivamente para estudiantes, se ha validado únicamente en los países pioneros, España y Holanda (9) y recientemente en México (6).

\section{Conclusiones}

Así se ha podido detectar que el engagement es útil ya que es el grado en que se comprometen los estudiantes con sus estudios, esto se caracteriza a través del vigor, destacando la energía al realizar un trabajo; la dedicación, siendo ésta, la importancia al trabajo y la absorción, considerando la concentración que empeña el estudiante en sus labores académicas. Estos elementos, son pilares para que la educación fluya de manera positiva en los estudiantes, evitando así un desgaste, que afecte su rendimiento académico.

En el engagement, la parte educativa deja de lado el bienestar del estudiante cómo persona, y se enfoca únicamente en su desempeño y la importancia de fomentar el mismo, ya que, las evidencias de su relación positiva con el bienestar tanto físico como psicológico logran que el engagement sea utilizado y reutilizado para el mejor aprovechamiento de los estudiantes. Adicionalmente, existen algunos autores que mencionan que, la contraparte del síndrome del burnout, es el engagement, el cual se fundamenta en la psicología positiva, desde este punto de vista, el concepto de engagement académico se asocia con los conceptos de vinculación psicológica o implicación a través del vigor, la dedicación y la absorción dedicada a los estudios $(7,9,10)$.

De igual manera, también se caracteriza por altos niveles de energía y una identificación con el trabajo (10), así como también influye en la lealtad hacia la institución donde realizan sus estudios.

\section{Referencias}

1. Portalanza Chavarría CA, Grueso Hinestroza MP, \& Duque Olivia, EJ. Propiedades de la Utrecht Work Engagement Scale (UWES-S 9): Análisis exploratorio con estudiantes en Ecuador Innovar. 2017:27 (64), 145-156.

2. Pérez MC, Molero MM, Barragán AB, Martos A, Simón MM, \& Gázquez JJ. Autoeficacia y engagement en estudiantes de Ciencias de la Salud y su relación con la autoestima. Publicaciones. 2018: 48(1), $161-172$.

3. Medrano LA, Moretti L, \& Ortiz A. Medición del Engagement Académico en Estudiantes Universitarios. Revista Iberoamericana de Diagnóstico y Evaluación - e Avaliação Psicológica. RIDEP. 2015: 40 (2), 114-123.

4. De la Cruz Pérez MA, \& Resendiz Chavez J. UWES-S México: Validación de la Utrecht Work Engagement Scale en Universitarios. Tesis de licenciatura. Universidad Autónoma del Estado de Hidalgo. México. 2017.

5. Cuevas Rivera F. Evaluación del engagement en empleados de una empresa comercial. Tesis de posgrado Universidad Autónoma de Nuevo León, México. 2011.

6. De la Cruz Pérez MA, Resendiz Chávez J, Romero Palencia A. \& Domínguez Aguirre GÁ. Adaptación y validación mexicana de la Utretch Work Engagement Scale, versión para estudiantes. Psicología Iberoamericana. 2017: 25(2), 35-43.

7. Oncu, S. Online Peer Evaluation for Assessing Perceived Academic Engagement in Higher Education.Journal of Mathematics, Science \& Technology Education. 2015:.11(3), 535-549.

8. King R, McInerney D, Ganotice F, \& Villarosa J. Positive affect catalyzes academic engagement: Cross-sectional, longitudinal, and experimental evidence. Learning and Individual Differences. 2015: 39, 64-72.

9. Caballero C, Hederich C, \& García A. Relación entre burnout y engagement académicos con variables sociodemográficas y académicas. Psicología desde el Caribe. 2015: 32(1), 254-267.

10 Schaufeli WB, Salanova M, González-Romá V, \& Bakker A. The Measurement of Burnout and Engagement: A Confirmatory Factor Analytic Approach. Journal of Happiness Studies. 2002: 3(1), 71-92.

11. Cavazos Arroyo J, \& Encinas Orozco FC. Influencia del engagement académico en la lealtad de estudiantes de posgrado: un abordaje a través de un modelo de ecuaciones estructurales. Estudios Gerenciales. 2016:, 32(140), 228-238. 